\title{
An Improved Interference Alignment Scheme for Frequency Selective Channels
}

\author{
Minqi Shen, Anders Høst-Madsen \\ Department of Electrical Engineering \\ University of Hawaii, Honolulu, HI 96822 \\ E-mail: $\{$ minqi,ahm\}@hawaii.edu.
}

\author{
Josep Vidal \\ Technical University of Catalonia \\ Dept. of Signal Theory and Communications, 08034 Barcelona SPAIN \\ E-mail: pepe@gps.tsc.upc.edu

$$
\begin{aligned}
\mathbf{y}^{j} & =\mathbf{H}_{j 1} \mathbf{x}^{1}+\mathbf{H}_{j 2} \mathbf{x}^{2}+\mathbf{H}_{j 3} \mathbf{x}^{3}+\cdots+\mathbf{H}_{j N} \mathbf{x}^{N}+\mathbf{z}^{j} \\
& =\mathbf{H}_{j j} \mathbf{x}^{j}+\left(\sum_{i=1, i \neq j}^{K} \mathbf{H}_{j i} \mathbf{x}^{i}\right)+\mathbf{z}^{j}
\end{aligned}
$$

\begin{abstract}
In this paper, the achievable rate of a K-user interference channel with single antenna at individual node is studied. Interference alignment and zero forcing are applied at the transmitter and receiver, respectively. It is well known from the paper [1] of Cadambe and Jafar that a multiplexing gain (degrees of freedom) of $K / 2$ is achievable. We propose two new pre-coding methods and study how they enhance the achievable rate in terms of the high SNR offset, while maintain the optimality of degree of freedom the Cadambe-Jafar scheme achieved.
\end{abstract}

\section{INTRODUCTION}

The degrees of freedom, or multiplexing gain, of a wireless channel with was introduced in [2]. It is defined as

$$
d_{f}=\lim _{\sigma^{2} \rightarrow 0} \frac{C\left(\sigma^{2}\right)}{\log \left(\sigma^{-2}\right)}
$$

where $\sigma^{2}$ is the noise power, and $C\left(\sigma^{2}\right)$ is the capacity of the channel. When SNR is large, $m$ becomes a basic character of the channel to describe the growth of data rate as a function of the SNR increment. Apparently, having a large $m$ is important for transmitting high speed data over a network.

The well known outerbound on degree of freedom for a $K$ user, single antenna interference system is $K / 2$, as presented in [3]. The paper [1] of Cadambe and Jafar shows the $K / 2$ degrees of freedom is achievable by deploying interference alignment at the transmitters and zero-forcing at the receivers, for systems with frequency selective channel. However, while the degree of freedom is optimized, how could the pre-coding matrices in an interference alignment scheme be designed so that the data rate will be maximized is still an open problem. In this paper, we relax the design constrains in [1], and propose two new pre-coding scheme. We will see that with some simple manipulation on the Cadambe-Jafar precoding matrices, the data rate could be improved, while the optimality of $d_{f}$ is maintained. One of the new pre-coding scheme is explicitly described and has higher data rate than the Cadambe-Jafar scheme with probability one.

\section{SySTEM MODEL}

Assume that the channel is comprised of $N$ orthogonal frequency dimensions. A K-user interference channel with a single antenna at individual node can be modeled as: where $i, j \in\{1,2, \cdots, K\}$

The length $N$ vector $\mathbf{y}^{j}$ is the signal received at the $j_{\text {th }}$ receiver; the length $N$ vector $\mathbf{x}^{i}$ is the coded message sent by the $i_{t h}$ transmitter; $\mathbf{H}_{j i}$ is a $N \times N$ diagonal matrix describing the channel between the $i_{t h}$ transmitter and the $j_{t h}$ receiver. Each diagonal entry $h_{i j}^{k}, k=1, \cdots, N$ is the fading coefficient of the $k_{t h}$ frequency slot, drawn independently from continuous random distribution. And the additive noise $\mathbf{z}^{j}$ is a length $N$ vector where every entry is i.i.d. (independent identically distributed) AWGN at the $j_{t h}$ receiver.

The $i_{t h}$ transmitter codes over all the orthogonal frequency dimensions. Then $\mathrm{x}^{i}$ can be described as:

$$
\mathbf{x}^{i}=\mathbf{V}_{i} \tilde{\mathbf{x}}^{i}
$$

where $\mathbf{V}_{i}$ is a $N \times d_{i}$ pre-coding matrix with rank $d_{i}$ and $\tilde{\mathbf{x}}^{i}$ is the original message vector with length $d_{i}$. We assume that $\mathbf{H}_{j i}$ and $\mathbf{V}_{i}$ are known a-priori at all nodes. And the $j_{t h}$ receiver wants to decode $\tilde{\mathbf{x}}^{j}$.

\section{INTERFERENCE ALIGNMENT AND ZERO-FORCING DECODING}

Interference alignment and zero-forcing decoding is a simple scheme fighting off interference. In an interference alignment and zero-forcing scheme, the message vector $\tilde{\mathbf{x}}^{i}$ is linearly transformed by a pre-codng matrix $\mathbf{V}_{i}$. With $\mathbf{V}_{i}$ designed properly, we could guarantee the message subspace and the interference subspace at receivers to be non-overlapping with probability one. Then the message could be obtained interference-free by zero forcing.

Zero forcing decoder works as follows. At the $j_{t h}$ receiver, the message subspace is spanned by the columns of $\mathbf{H}_{j j} \mathbf{V}_{j}$; the interference subspace is spanned by the columns of $\mathbf{H}_{j i} \mathbf{V}_{i}, i \neq j$. Let $\mathbf{S}_{j}^{\perp}$ be the basis spanning the orthogonal 
complement of the interference subspace $\mathbf{S}_{j}$. A zero-forcing receiver transforms $\mathbf{y}^{j}$ by $\mathbf{S}_{j}^{\perp}$ :

$$
\hat{\mathbf{y}}^{j}=\left(\mathbf{S}_{j}^{\perp}\right)^{\prime} \mathbf{y}^{j}=\left(\mathbf{S}_{j}^{\perp}\right)^{\prime} \mathbf{H}_{j j} \mathbf{V}_{j} \tilde{\mathbf{x}}^{j}+\left(\mathbf{S}_{j}^{\perp}\right)^{\prime} \mathbf{z}^{j}
$$

where $\tilde{\mathbf{x}} \sim N\left(0, \rho \mathbf{I}_{d_{i} \times d_{i}}\right), \tilde{\mathbf{z}} \sim \mathrm{N}\left(0, \mathbf{I}_{N \times N}\right)$, where the power of $\mathbf{V}_{i}$ is normalized to 1: $\frac{1}{N} \operatorname{trace}\left(\mathbf{V}_{i} \mathbf{V}_{i}^{\prime}\right)=1$. Further, if $d_{s_{j}^{\perp}}=d_{j}$, then $\left(\mathbf{S}_{j}^{\perp}\right)^{\prime} \mathbf{H}_{j j} \mathbf{V}_{j}$ is invertible, and the rate of $j_{t} h$ user is

$$
\begin{aligned}
R_{j}= & \log \left(\left|\mathbf{I}_{d_{j} \times d_{j}}+\rho \mathbf{K}_{\tilde{\mathbf{z}}^{j}}^{-1}\right|\right) \\
= & \log \left(\mid \mathbf{I}_{d_{j} \times d_{j}}+\rho\left(\mathbf{H}_{j j} \mathbf{V}_{j}-\mathbf{P}_{S_{j}}\left(\mathbf{H}_{j j} \mathbf{V}_{j}\right)\right)^{\prime}\right. \\
& \left.\left(\mathbf{H}_{j j} \mathbf{V}_{j}-\mathbf{P}_{S_{j}} \cdot\left(\mathbf{H}_{j j} \mathbf{V}_{j}\right)\right) \mid\right)
\end{aligned}
$$
where $\mathbf{K}_{\tilde{\mathbf{z}}^{j}}=\left(\left(\left(\mathbf{S}_{j}^{\perp}\right)^{\prime} \mathbf{H}_{j j} \mathbf{V}_{j}\right)^{-1}\left(\mathbf{S}_{j}^{\perp}\right)^{\prime}\right)\left(\left(\left(\mathbf{S}_{j}^{\perp}\right)^{\prime} \mathbf{H}_{j j} \mathbf{V}_{j}\right)^{-1}\left(\mathbf{S}_{j}^{\perp}\right)^{\prime}\right)^{\mathfrak{a})}$, is equivalent with (c):

$\mathbf{P}_{S}$ is the projection matrix for subspace $S_{j}$.

Define the sum rate as $R=\frac{\left(\sum_{j=1}^{K} R_{j}\right)}{N}$ and the degree of freedom $d=\frac{\sum_{j=1}^{K} d_{j}}{N}$. (5) shows the high snr offset [5], [6], [4]in a system using interference alignment and zero-forcing.

\section{The Cadambe-Jafar Scheme And Possible IMPROVEMENTS FOR 3-USER SYSTEM}

[1] proposed a precoding scheme achieving $((n+$ $\left.1)^{N}, n^{N}, n^{N}, \cdots, n^{N}\right)$ degree of freedom over a symbol extension of $(n+1)^{N}+n^{N}, N=(K-1)(K-2)-1$, which implies that

$$
\max _{\left(d_{1}, d_{2}, \cdots, d_{K}\right) \in D} \geq \sup _{n} \frac{(\mathrm{n}+1)^{\mathrm{N}}+(\mathrm{K}-1) \mathrm{n}^{\mathrm{N}}}{(\mathrm{n}+1)^{\mathrm{N}}+\mathrm{n}^{\mathrm{N}}}=\mathrm{K} / 2
$$

Further, this degree of freedom could be achieved by interference-alignment and zero-forcing. The achievability proof is broken into two parts. Part one states that given perfect channel knowledge, there exists a precoding scheme in which the rank of interference space at $K$ receivers is $\left(n^{N},(n+1)^{N},(n+1)^{N}, \cdots,(n+1)^{N}\right)$. Part two proves that using this scheme, the received signal spanning full rank $\left((n+1)^{N}+n^{N}\right)$ at each receiver, so that the message subspace and the interference subspace are non-overlapping. The key for part 2 is that the fading coefficients are drawn from continues random distribution.

In the rest of this paper, we discuss two perspective to improve the Cadambe-Jafar scheme: one is trying to find better precoding subspaces; the other optimizes precoding vectors within the subspaces constructed by Cadambe-Jafar. While the first problem could be complicated, we will see that from the second perspective, the sum rate can be improved by orthonomalizing the precoding vectors at each transmitter.

First, we consider the case $K=3, N=2 n+1, n>0, n \in Z$. If and only if the pre-coding matrices satisfying condition (a):

$$
\left\{\begin{array}{l}
\operatorname{span}\left(\mathbf{H}_{12} \mathbf{V}_{2}\right)=\operatorname{span}\left(\mathbf{H}_{13} \mathbf{V}_{3}\right) \\
\operatorname{span}\left(\mathbf{H}_{23} \mathbf{V}_{3}\right) \subset \operatorname{span}\left(\mathbf{H}_{21} \mathbf{V}_{1}\right) \\
\operatorname{span}\left(\mathbf{H}_{32} \mathbf{V}_{2}\right) \subset \operatorname{span}\left(\mathbf{H}_{31} \mathbf{V}_{1}\right)
\end{array}\right.
$$

and (b):

$$
\left\{\begin{array}{l}
\operatorname{span}\left(\mathbf{H}_{11} \mathbf{V}_{1}\right) \cap \operatorname{span}\left(\mathbf{H}_{12} \mathbf{V}_{2}\right)=\emptyset \\
\operatorname{span}\left(\mathbf{H}_{22} \mathbf{V}_{2}\right) \cap \operatorname{span}\left(\mathbf{H}_{21} \mathbf{V}_{1}\right)=\emptyset \\
\operatorname{span}\left(\mathbf{H}_{33} \mathbf{V}_{3}\right) \cap \operatorname{span}\left(\mathbf{H}_{31} \mathbf{V}_{1}\right)=\emptyset
\end{array}\right.
$$

\section{$\Downarrow$}

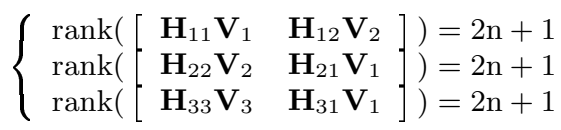

a scheme could achieve $\frac{3 n+1}{2 n+1}$ degree of freedom in a system described by (1) using zero-forcing receivers. [1] proved that

$$
\left\{\begin{array}{l}
\operatorname{span}(\mathbf{B})=\operatorname{span}\left(\mathbf{T}_{1} \mathbf{C}\right) \\
\operatorname{span}(\mathbf{B}) \subset \operatorname{span}(\mathbf{A}) \\
\operatorname{span}(\mathbf{C}) \subset \operatorname{span}(\mathbf{A})
\end{array}\right.
$$

where $\left\{\begin{array}{l}\mathbf{A}=\tilde{\mathbf{V}}_{1} \\ \mathbf{B}=\left(\mathbf{H}_{21}\right)^{-1} \mathbf{H}_{23} \tilde{\mathbf{V}}_{3} \\ \mathbf{C}=\left(\mathbf{H}_{31}\right)^{-1} \mathbf{H}_{32} \tilde{\mathbf{V}}_{2} \\ \mathbf{T}_{1}=\mathbf{H}_{12}\left(\mathbf{H}_{21}\right)^{-1} \mathbf{H}_{23}\left(\mathbf{H}_{32}\right)^{-1} \mathbf{H}_{31}\left(\mathbf{H}_{13}\right)^{-1}\end{array}\right.$

By restricted (c) into $(\mathrm{d})$ :

$$
\left\{\begin{array}{l}
\mathbf{B}=\mathbf{T}_{1} \mathbf{C} \\
\mathbf{B} \prec \mathbf{A} \\
\mathbf{C} \prec \mathbf{A}
\end{array}\right.
$$

the Cadambe-Jafar scheme is:

$$
\left\{\begin{array}{l}
\mathbf{A}=\left[\begin{array}{lllll}
\mathbf{w} & \mathbf{T}_{1} \mathbf{w} & \mathbf{T}_{1}^{2} \mathbf{w} & \ldots & \mathbf{T}_{1}^{n} \mathbf{w}
\end{array}\right] \\
\mathbf{B}=\left[\begin{array}{lllll}
\mathbf{T}_{1} \mathbf{w} & \mathbf{T}_{1}^{2} \mathbf{w} & \ldots & \mathbf{T}_{1}^{n} \mathbf{w}
\end{array}\right] \\
\mathbf{C}=\left[\begin{array}{lllll}
\mathbf{w} & \mathbf{T}_{1} \mathbf{w} & \ldots & \mathbf{T}_{1}^{n-1} \mathbf{w}
\end{array}\right]
\end{array}\right.
$$

where $\mathbf{w}$ is all-one vector, $\mathbf{V}_{1}, \mathbf{V}_{2}$ and $\mathbf{V}_{3}$ are $(2 n+1) \times(n+$ $1),(2 n+1) \times n$, and $(2 n+1) \times n$ full-column rank matrices respectively. While (d) is apparently satisfied, [1] proved that (b) is true with probability one. To consider cases where snr $<$ $\infty$, we normalize the power of $\mathbf{V}_{i}$ to 1 .

\section{A. Designing vector $\mathbf{w}$}

It could be proved that as long as $\mathbf{w}$ has no zero entry, the Cadambe-Jafar scheme will achieve $\frac{3 n+1}{2 n+1}$ degree of freedom. According to (5),

$$
R-D \log (s n r)=\frac{\log \left(f\left(w_{1}, w_{2}, \cdots, w_{N}\right)\right)}{N}+2
$$

where $\quad f\left(w_{1}, w_{2}, \cdots, w_{N}\right)$

$$
\prod_{j=1}^{K}\left|\left(\mathbf{H}_{j j} \mathbf{V}_{j}-\mathbf{P}_{S_{j}}\left(\mathbf{H}_{j j} \mathbf{V}_{j}\right)\right)^{\prime}\left(\mathbf{H}_{j j} \mathbf{V}_{j}-\mathbf{P}_{S_{j}}\left(\mathbf{H}_{j j} \mathbf{V}_{j}\right)\right)\right|
$$

$f\left(w_{1}, w_{2}, \cdots, w_{N}\right)$ is a bounded and continuous function of $w_{i}$ for $w_{i} \neq 0$. But as $f\left(w_{1}, w_{2}, \cdots, w_{N}\right)$ is not defined at $w_{i}=0$, the Wierstrass theorem is not satisfied, there might not exist a close-form optimal solution that maximizing the high SNR off set. And the expression could be very complicated as $N$ growing larger. Fig 1 shows in an example of how different $\mathbf{w}$ could give different transmitting rate, with $N=3$. 
$\lim _{s n r \rightarrow \infty} R-D \log (s n r)=\frac{\log \left(\prod_{j=1}^{K}\left|\left(\mathbf{H}_{j j} \mathbf{V}_{j}-\mathbf{P}_{S_{j}}\left(\mathbf{H}_{j j} \mathbf{V}_{j}\right)\right)^{\prime}\left(\mathbf{H}_{j j} \mathbf{V}_{j}-\mathbf{P}_{S_{j}}\left(\mathbf{H}_{j j} \mathbf{V}_{j}\right)\right)\right|\right)}{N}$

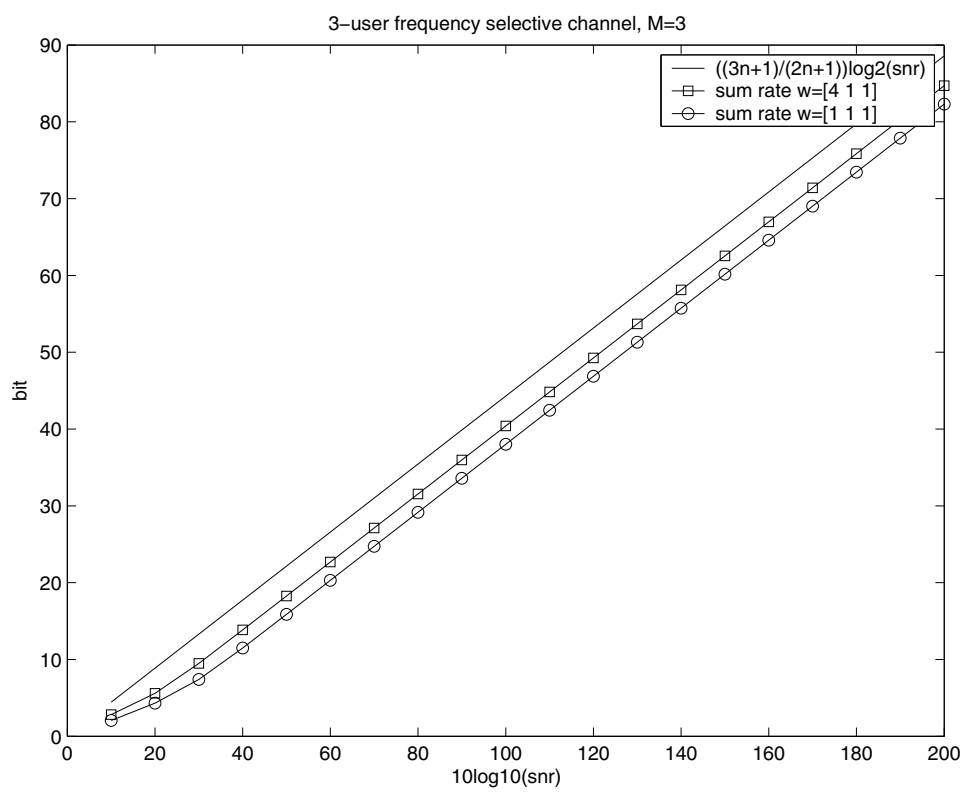

Fig. 1. Sum-rate vs. SNR under different w vectors

\section{B. Another Extended Design}

(c) is equivalent with (e):

$$
\left\{\begin{array}{l}
\mathbf{B}=\mathbf{T}_{1} \mathbf{C} \cdot \mathbf{D} \\
\mathbf{B}=\mathbf{A}_{b} \cdot \mathbf{E} \\
\mathbf{C}=\mathbf{A}_{c} \cdot \mathbf{F}
\end{array}\right.
$$

where $\mathbf{A}_{b}$ and $\mathbf{A}_{c}$ are matrices composed by $n$ columns chosen out of $n+1$ columns of $\mathbf{A}$. D, $\mathbf{E}$ and $\mathbf{F}$ are non-singular $n \times n$ matrices.

Following the C-J scheme, we fix $\mathbf{w}$ to be $\tilde{\mathbf{1}}$, and

$$
\begin{gathered}
\mathbf{A}=\left[\begin{array}{lllll}
\mathbf{w} & \mathbf{T}_{1} \mathbf{w} & \cdots & \mathbf{T}_{1}^{n-1} \mathbf{w} & \mathbf{T}_{1}^{n-1} \mathbf{w}
\end{array}\right] \\
\mathbf{A}_{b}=\left[\begin{array}{llll}
\mathbf{w} & \mathbf{T}_{1} \mathbf{w} & \cdots & \mathbf{T}_{1}^{n-1} \mathbf{w}
\end{array}\right]
\end{gathered}
$$$$
\mathbf{A}_{c}=\left[\begin{array}{llll}
\mathbf{T}_{1} \mathbf{w} & \mathbf{T}_{1}^{2} \mathbf{w} & \cdots & \mathbf{T}_{1}^{n} \mathbf{w}
\end{array}\right]
$$

To find maximum high SNR off set, we want to solve the following problem:

$$
\begin{aligned}
& \max \prod_{j=1}^{K} p_{j}\left|\left(\mathbf{H}_{j j} \mathbf{V}_{j}\right)^{\prime}\left(\mathbf{H}_{j j} \mathbf{V}_{j}\right)\right| \\
& \text { s.t. } \frac{1}{N} \operatorname{trace}\left(\tilde{\mathbf{V}}_{i} \tilde{\mathbf{V}}_{i}^{\prime}\right)=1 \\
& \mathbf{V}_{1}=\tilde{\mathbf{V}}_{1}\left[\frac{1}{\frac{1}{N} \operatorname{trace}\left(\tilde{\mathbf{V}}_{1} \tilde{\mathbf{V}}_{1}^{\prime}\right)}\right]^{\frac{1}{2}} \\
& \mathbf{V}_{2}=\tilde{\mathbf{V}}_{2} \mathbf{F} \\
& \mathbf{V}_{3}=\tilde{\mathbf{V}}_{3} \mathbf{E} \\
& \mathbf{w}=\mathbf{1} \\
& \mathbf{T}_{1}=\mathbf{H}_{12}\left(\mathbf{H}_{21}\right)^{-1} \mathbf{H}_{23}\left(\mathbf{H}_{32}\right)^{-1} \mathbf{H}_{31}\left(\mathbf{H}_{13}\right)^{-1} \\
& \mathbf{T}_{2}=\left(\mathbf{H}_{32}\right)^{-1} \mathbf{H}_{31} \\
& \mathbf{T}_{3}=\left(\mathbf{H}_{23}\right)^{-1} \mathbf{H}_{21} \\
& \tilde{\mathbf{V}}_{1}=\left[\begin{array}{lllll}
\mathbf{w} & \mathbf{T}_{1} \mathbf{w} & \mathbf{T}_{1}^{2} \mathbf{w} & \cdots & \mathbf{T}_{1}^{n} \mathbf{w}
\end{array}\right] \\
& \tilde{\mathbf{V}}_{2}=\mathbf{T}_{2}\left[\begin{array}{llll}
\mathbf{w} & \mathbf{T}_{1} \mathbf{w} & \cdots & \mathbf{T}_{1}^{n-1} \mathbf{w}
\end{array}\right] \\
& \tilde{\mathbf{V}}_{3}=\mathbf{T}_{3}\left[\begin{array}{llll}
\mathbf{T}_{1} \mathbf{w} & \mathbf{T}_{1}^{2} \mathbf{w} & \ldots & \mathbf{T}_{1}^{n} \mathbf{w}
\end{array}\right] \\
& \mathbf{F}, \mathbf{E} \text { are non }- \text { singular matrices, } \mathbf{F D}=\mathbf{E}
\end{aligned}
$$

where $p_{j}$ is a positive projection constant at receiver $\mathrm{j}$. Because

1) $\left|\left(\mathbf{H}_{22} \tilde{\mathbf{V}}_{2} \mathbf{F}\right)^{\prime}\left(\mathbf{H}_{22} \tilde{\mathbf{V}}_{2} \mathbf{F}\right)\right|=|\mathbf{F}|^{2}\left|\left(\mathbf{H}_{22} \tilde{\mathbf{V}}_{2}\right)^{\prime}\left(\mathbf{H}_{22} \tilde{\mathbf{V}}_{2}\right)\right|$, where $\left|\left(\mathbf{H}_{22} \tilde{\mathbf{V}}_{2}\right)^{\prime}\left(\mathbf{H}_{22} \tilde{\mathbf{V}}_{2}\right)\right|$ is a constant determined by the channel, 
2) A matrix $\mathbf{F}$ maximizing $|\mathbf{F}|^{2}$ also maximizes $\left|\left(\tilde{\mathbf{V}}_{2} \mathbf{F}\right)^{\prime}\left(\tilde{\mathbf{V}}_{2} \mathbf{F}\right)\right|$, Our problem could be further simplified into maximizing $\left|\left(\tilde{\mathbf{V}}_{2} \mathbf{F}\right)^{\prime}\left(\tilde{\mathbf{V}}_{2} \mathbf{F}\right)\right|$ and $\left|\left(\tilde{\mathbf{V}}_{3} \mathbf{E}\right)^{\prime}\left(\tilde{\mathbf{V}}_{3} \mathbf{E}\right)\right|$ separately under the power constrain $\frac{1}{N} \operatorname{trace}\left(\left|\left(\tilde{\mathbf{V}}_{2} \mathbf{F}\right)^{\prime}\left(\tilde{\mathbf{V}}_{2} \mathbf{F}\right)\right|\right)=1$ and $\frac{1}{N} \operatorname{trace}\left(\left|\left(\tilde{\mathbf{V}}_{3} \mathbf{E}\right)^{\prime}\left(\tilde{\mathbf{V}}_{3} \mathbf{E}\right)\right|\right)=1$.

The maximum solution will be obtained when the columns of $\mathbf{V}_{i}$ are orthogonal, each has power of $\frac{N}{d_{i}}$. Thus, the optimal $\mathbf{E}$ and $\mathbf{F}$ are $n \times n$ matrices that orthonormalizing $\quad \mathbf{T}_{3}\left[\begin{array}{llll}\mathbf{T}_{1} \mathbf{w} & \mathbf{T}_{1}^{2} \mathbf{w} & \ldots & \mathbf{T}_{1}^{n} \mathbf{w}\end{array}\right] \quad$ and $\mathbf{T}_{2}\left[\begin{array}{llll}\mathbf{w} & \mathbf{T}_{1} \mathbf{w} & \ldots & \mathbf{T}_{1}^{n-1} \mathbf{w}\end{array}\right]$, respectively.

Since the Cadambe-Jafar scheme is a special case of our new scheme, with $\mathbf{D}=\mathbf{E}=\mathbf{F}=$ IdentityMatrix, we could expect that the new scheme out-perform the Cadambe-Jafar scheme with probability one. Fig 2 shows the simulation result of $R-R_{0}$ under the high snr assumption, for 9 frequency slots. Fading coefficients are drawn i.i.d from standard Gaussian distribution. Further, when SNR goes to infinite, the increment in sum-rate over that of the Cadambe-Jafar scheme is a constant that only depends on the channel and pre-coding matrices. Fig 3 compares the Sum-rate vs. SNR plots of the new scheme and that of the Cadambe-Jafar scheme. Notation 0 represent the parameters in the Cadambe-Jafar scheme.

\section{K-user, Even Frequency Slots System}

Notice that because $\mathbf{E}$ and $\mathbf{F}$ are optimized independently, and the Cadambe-Jafar scheme has similar precoding matrices structure for $K>3$, even number of frequency slots system, this new scheme could be extended into more general cases.

\section{CONCLUSION}

In this paper, we have explored two perspective to improve the Cadambe-Jafar interference alignment scheme, which achieve $K / 2$ degree of freedom in a $K$ user interference system with frequency selective channel. One is trying to find better precoding subspaces; the other optimizes precoding vectors within the subspaces constructed by Cadambe-Jafar scheme. We fail to give explicit solution for the first problem, though simulation result shows that the data rate could be improved when different $\mathrm{w}$ vector is chosen. For the second problem, we prove that choosing orthonormal pre-coding matrices to construct the transmitting-data subspace of CadambeJafar scheme will increase the sum rate with probability one, without hurting the $K / 2$ degree of freedom.

\section{REFERENCES}

[1] Viveck R. Cadambe and Syed A. Jafar. "Interference Alignment and the Degrees of Freedom for the $\mathrm{K}$ User Interference Channel". arXiv: 0707.0323, e-print. available at http://newport.eecs.uci.edu/ syed/papers/intK.pdf

[2] L. Zheng and D. Tse "Diversity and multiplexing: A fundamental tradeoff in multiple antenna channels". In IEEE Trans. on Inf. Theory, 2002.

[3] A. Høst-Madsen and A. Nosratinia "The multiplexing gain of wireless networks". In Proceedings of IEEE International Symposium on Information Theory: ISIT'05 (Adelaide, Australia), 2005.
[4] N. Jindal. "High snr analysis of mimo broadcast channels". In Proceedings of IEEE International Symposium on Information Theory: ISIT'05 (Adelaide, Australia), 2005.

[5] A. Lozano, A.M. Tulino, and S. Verdu. "High-snr power offset in multiantenna communication". IEEE Transactions on Information Theory, 51(12):4134 - 4151, December 2005.

[6] S. Shamai and S. Verdu. "The impact of frequency-flat fading on the spectral efficiency of cdma". IEEE Transactions on Information Theory, 47(4):1302 - 1327, May 2001 


$$
\lim _{s n r \rightarrow \infty} R-R_{0}=(2 n+1)^{-1}\left(2 n \log \left(\frac{2 n+1}{n}\right)+\log \left(\left|\mathbf{V}_{02}^{\prime} \mathbf{V}_{02}\right|^{-1}\left|\mathbf{V}_{03}^{\prime} \mathbf{V}_{03}\right|^{-1}\right)\right)
$$

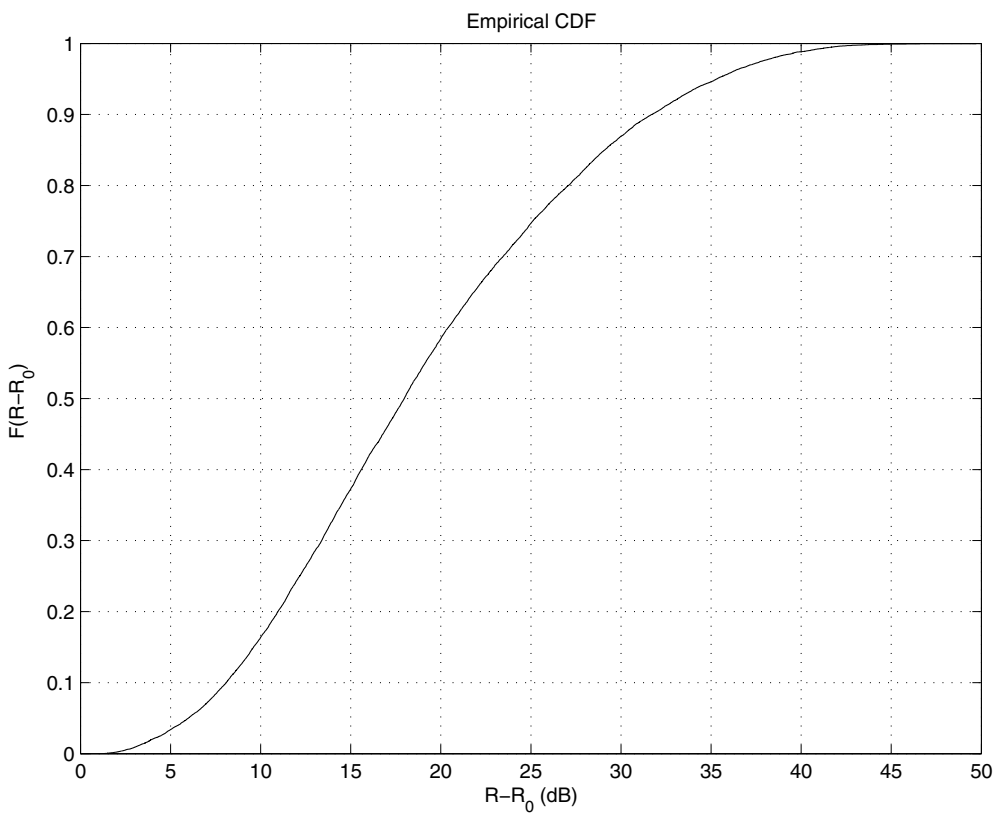

Fig. 2. CDF of the Sum rate difference between two schemes

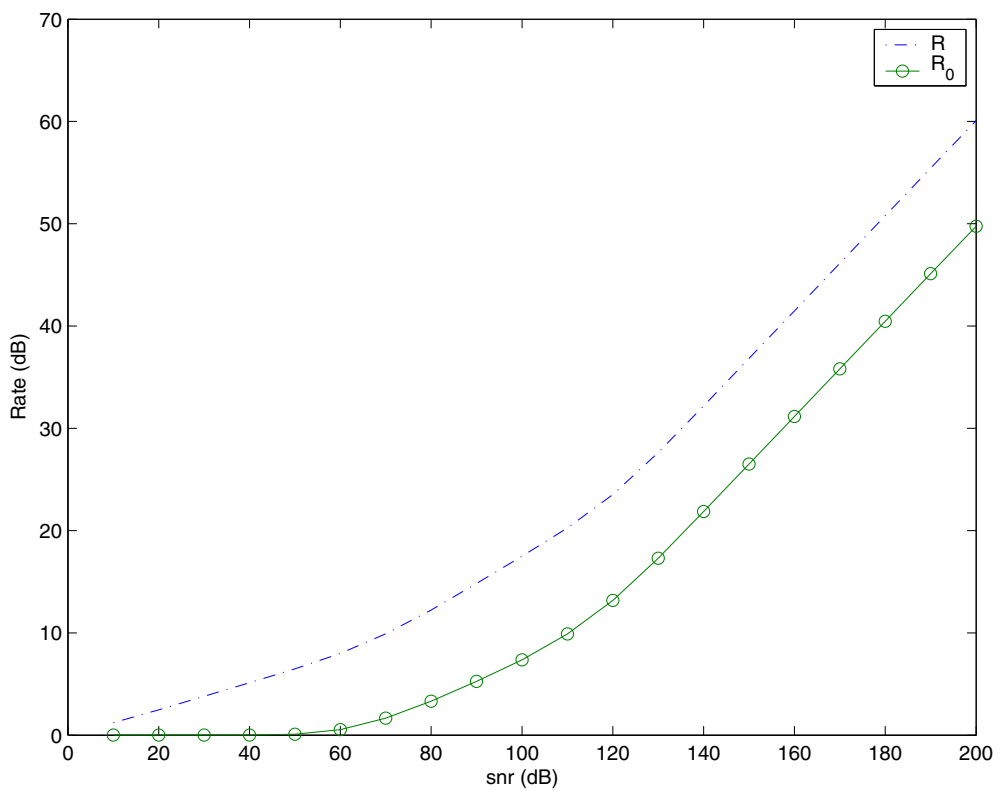

Fig. 3. Comparison of Sum rate vs. SNR 